\title{
Caisse de secours des médecins suisses
}

\author{
Rapport annuel 2004
}

Correspondance: Dr U. Leibundgut Président de la Commission de fondation Lange Gasse 78 CH-4052 Bâle
Au cours de l'année sous revue, la Caisse de secours des médecins suisses a accordé son soutien à 31 médecins tombés malgré eux dans le besoin (ils étaient 26 en 2003), ainsi qu'aux proches et descendants de consœurs et de confrères décédés.

Pendant la même période, la Caisse a cessé d'apporter son aide à trois personnes, pour cause de décès. Toujours en 2004, quatre nouveaux cas sont venus s'ajouter à la liste des bénéficiaires: trois personnes ont obtenu une aide unique et une personne nécessitera probablement un soutien pendant une longue période.

La somme globale des contributions versées s'est élevée en 2004 Fr. 318900.- contre Fr. 264 200.- l'année précédente.

Les recettes en provenance de dons, d'un montant de Fr. 128573.85 (2003: 157 365.70), ont malheureusement diminué à nouveau en 2004; il en va de même pour le rendement du capital, d'un montant de Fr. 57234.78 (2003: $69759.31)$. Ces recettes directes correspondent aux $45 \%$ des charges de la Fondation, qui ont atteint un total de Fr. 411185.78 (2003: $320103.16)$. L'équilibre du compte d'exploitation n'a ainsi pu être obtenu qu'avec la dissolution de provisions, d'un montant de Fr. 51 000.-, et par un recours supplémentaire à la fortune, qui s'est réduite de Fr. 173900.25.

$\mathrm{Au} 31$ décembre 2004, la fortune de la fondation s'élevait à Fr. 2493463.59 (2003: Fr. 2673 749.39). Vu la diminution des recettes provenant de dons et du rendement du capital, les pertes enregistrées sur le marché des capitaux et les charges simultanément plus élevées pour les aides, la fortune a diminué de Fr. 180 285.80.

La fortune de la fondation est constituée par un mélange d'obligations et d'actions satisfaisant aux prescriptions de la prévoyance professionnelle (LPP). Le compte d'exploitation, le bilan et la gestion de la fortune sont révisés chaque année par l'autorité de surveillance des fondations du Département de justice du canton de Bâle-Ville.
Les dons individuels inférieurs à Fr. 500.- forment l'essentiel du capital de notre fondation. Par souci de réduire les frais administratifs au strict minimum, nous renonçons toutefois à remercier personnellement les donateurs. Qu'ils veuillent trouver ici l'expression de notre vive gratitude!

MM. F. Hufschmid et R. Jucker, réviseurs, ont examiné les comptes annuels de la Caisse de secours et constaté que la comptabilité était tenue dans les règles. Nous saisissons l'occasion pour leur exprimer nos vifs remerciements. Pour plus de détails, le lecteur se référera au compte d'exploitation et au bilan publiés ci-après.

Nos sincères remerciements vont également à MM. les Drs Ludwig Heuss et W. Zutter ainsi qu'à MM. J. Kobler et W. Mahrer de la Banque La Roche \& Co pour la tenue de la comptabilité et la gestion de la fortune.

La Caisse de secours des médecins suisses a été fondée il y a plus de 100 ans. Le siècle dernier a vu la création et le développement de nombreuses institutions de prévoyance et d'assurance publiques et privées. Malgré ces institutions, il y a encore aujourd'hui des personnes durement frappées par le sort et non secourues par les institutions connues. Seule l'aide privée est en mesure de leur procurer un soutien. La caisse de secours des médecins suisses compte sur vos contributions, au $21^{\mathrm{e}}$ siècle également!

Le Conseil de fondation de la Caisse de secours des médecins suisses:

Dr U. Leibundgut, président

Dr L. T. Heuss, membre du Comité central de la FMH

DrW. Zutter, trésorier

Caisse de secours des médecins suisses: CCP 40-644-3 Bâle. 